\title{
CURRENT-DRIVEN QUANTUM DYNAMICS OF TOROIDAL MOMENT IN RARE-EARTH NANOCLUSTERS
}

\author{
D.I. Plokhov ${ }^{1, \star}$, A.I. Popov ${ }^{2,3}$, and A.K. Zvezdin ${ }^{1,2}$ \\ ${ }^{1}$ A.M. Prokhorov General Physics Institute, Russian Academy of Sciences, Moscow, Russia \\ ${ }^{2}$ Moscow Institute of Physics and Technology, Dolgoprudny, Moscow Region, Russia \\ ${ }^{3}$ National Research University of Electronic Technology, Zelenograd, Moscow, Russia
}

\begin{abstract}
The current-driven quantum dynamics of toroidal moment in single molecule magnets with polygonal rare-earth ion core is investigated. The effects of an external current is considered both in equilibrium and in the frames of the Landau-Zener-Stückelberg tunneling model. It is shown that the toroidal moment is a suitable degree of freedom for using the systems in question as qubits in quantum computing.
\end{abstract}

\section{Introduction}

The idea of quantum computation began to be actively discussed in the world in 1982, after the publication of R. Feynman [1]. The avalanche-like growth in the number of publications on quantum computation was caused by Shor's article [2] on quantum (implemented on a quantum computer) algorithms. Further progress in the area of quantum computing mainly follows two lines, the development and improvement of quantum algorithms and the search for suitable physical implementations of a qubit. The basic requirements (so called DiVincenzo checklist) for physical systems claiming the role of qubits are formulated in ref. [3].

Over last two decades, several physical implementations of a qubit have been proposed. Some of them origin from quantum optics: cavity quantum electrodynamics [4], including linear optic quantum computation [5] with the so-called boson sampling [6]. Others deal with ultracold atoms: trapped ion quantum computer [7] and optical lattice one [8]. Very exciting are spin-based representations: Kane quantum computer [9], Loss-DiVincenzo quantum computer [10], and nitrogen-vacancy center quantum computer [11]. Quite elaborated are superconducting qubits: charge, flux, and phase [12]. There are also proposals $[13,14]$ on the use of magnetic particles in quantum computing.

There has been made a great progress on the way, but the «ideal» qubit has not been developed yet. Research efforts are focused mainly on the properties of the very physical systems. At the same time, the search for new implementations still remains topical. We propose to use a new degree of freedom, the toroidal (anapole) moment, which is natural characteristics of recently synthesized family of dysprosium based ring single molecule magnets (toroics). The toroidal moment is easily accessible by electric means

\footnotetext{
^e-mail: dmitry.plokhov@gmail.com
}

(electric fields, which are more localizable than magnetic ones, or just an electric current). In this paper, we describe the macroscopic quantum dynamics of the toroidal moment coupled with an electric current. The results make it possible to conclude that toroidal moment is convenient to implement an anapole qubit. The compliance with the DiVincenzo criteria is discussed.

\section{Single molecule toroics}

We consider a spin ring, a system of $N$ non-Kramers rareearth ions located in the apices of a regular polygon. The ground state of rare-earth ions in compounds is formed mainly due to the influence of a crystal field and often has a strong anisotropy of the magnetic moment. For example, the ground state of $\mathrm{Dy}^{3+}$ ions in $\mathrm{Dy}_{3}$ cluster is the Kramers doublet $\left|M_{J}= \pm 15 / 2\right\rangle$ [15] and responds only to the $z_{i}$ local component of an external magnetic field (see fig. 1). The first excited states are close to $\left|M_{J}= \pm 13 / 2\right\rangle$ and separated from the ground one by the gap of $200 \mathrm{~cm}^{-1}$. In the case of non-Kramers ions, asymmetrical perturbations remove degeneration, thus making the ground state be quasi-doublet, i.e. the two close singlets with splitting small compared with distance from the higher levels. The effective Hamiltonian of the system reads

$$
\mathcal{H}=-\frac{j}{4} \sum_{i<k}^{N} \sigma_{i z} \sigma_{k z}-\frac{\Delta}{2} \sum_{i=1}^{N} \sigma_{i x}-g_{J} \mu_{B} \sum_{i=1}^{N} H_{z_{i}} J_{z_{i}} \sigma_{i z}
$$

where $\sigma_{x}, \sigma_{y}$, and $\sigma_{z}$ stand for the Pauli matrices, $j$ is the exchange interaction constant $\left(j \sim 10 \mathrm{~cm}^{-1}\right), \Delta$ is the energy gap between the singlet levels of an ion in the crystal field with respect to the local axes $\left(\Delta \sim 0.05 \mathrm{~cm}^{-1}\right)$. It is shown [16] that eq. (1) is valid not only for non-Kramers ions, but for Kramers ions as well. In the latter case, the splitting $\Delta$ can be produced by an external magnetic field $H$, and $\Delta \rightarrow 0$ at $H \rightarrow 0$. 


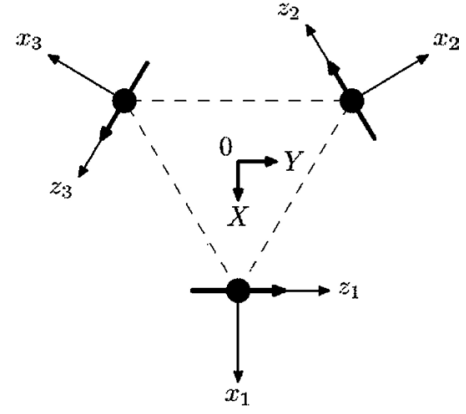

Figure 1. The spin structure of a triangular rare-earth molecular cluster and the local easy axes orientation in respect of the laboratory $X Y Z$ reference frame. The thicker arrows represent the spins of the rare-earth ions in the molecule in the state with toroidal moment $T_{Z}=+3 T_{0}\left(T_{0}=\frac{1}{2} g_{J} \mu_{B} r_{0} J\right)$.

There are $2^{N}$ possible orderings of the ion spins in the rare-earth polygonal cluster. The spin orderings can be characterized in terms of spin chirality. It is clear that there are always pairs of states with spins inversely twisted, i.e. the states have opposite chirality. The natural physical quantity associated with spin chirality in this case is the $T$ odd polar vector of the anapole (toroidal) moment, so the systems in question can be called single molecule toroics (SMTs) by analogy of single molecule magnets (SMMs). We would remind that the toroidal moment operator in the case of localized magnetic ions can be defined as

$$
\mathbf{T}=\frac{1}{2} g_{J} \mu_{B} \sum_{i=1}^{N}\left[\mathbf{r}_{i} \times \mathbf{J}_{i}\right]
$$

where $\mathbf{r}_{i}$ is the radius-vector connecting the center of the $N$-gon with the $i$-th apex (all $r_{i}=r_{0}$ ). The toroidal moment of the system is just formally equal to the effective "magnetization", associated with the effective Hamiltonian in eq. (1). The values of the toroidal moments for conjugate states are different in sign.

\section{The current driven dynamics}

To describe the interaction between the toroidal moment and an external electric current it is necessary to put the term $\hat{V}=\frac{4 \pi}{c} \mathbf{j} \hat{\mathbf{T}}$ into the Hamiltonian in eq. (1). The electric current $\mathbf{j}$ could be produced as the displacement current $\mathbf{j}=\frac{1}{4 \pi} \frac{\partial \mathbf{E}}{\partial t}$. If the electric field strength $\mathbf{E}$ linearly depends on the time and is directed along the $Z$-axis, then $\hat{V}=\frac{v}{c}$. $\hat{T}$, where $v=\partial E_{Z} / \partial t$. The energies of the two low-lying levels could be approximated in the vicinity of the avoided level crossing at $j_{z}=0$ as (see ref. [16])

$$
E_{ \pm}\left(x, j_{z}\right)=E_{0} \mp \sqrt{\left(\frac{4 \pi}{c} \cdot 3 T_{0} \cdot j_{z}\right)^{2}+\left(\frac{\delta}{2}\right)^{2}},
$$

where the crossing energy is $E_{0}=-\frac{3}{4} j$, the avoided level splitting is $\delta=\frac{3}{2} j x^{3}, x=\Delta / j$, and $T_{0}=\frac{1}{2} g_{J} \mu_{B} r_{0} J$.

The toroidal moment is a conjugate variable against the electric current density and can be found at zero tem- perature as

$$
T(x)=\frac{c}{4 \pi} \frac{\partial E_{-}\left(x, j_{z}\right)}{\partial j_{z}}, \quad \tau(x)=\frac{T(x)}{T_{0}} .
$$

The plot of the relative (equilibrium) dependence is given in fig. 2 (curve 1). It is seen, that the current changes the direction of the spin twisting to the opposite, thus reversing the toroidal moment of the system in a way of the relatively sharp jump of $\Delta \tau=6$. The anapole moment reversal, which could be called reanapolization, the reanapolization requires the currents of $10^{7} \mathrm{~A} / \mathrm{cm}^{2}$.

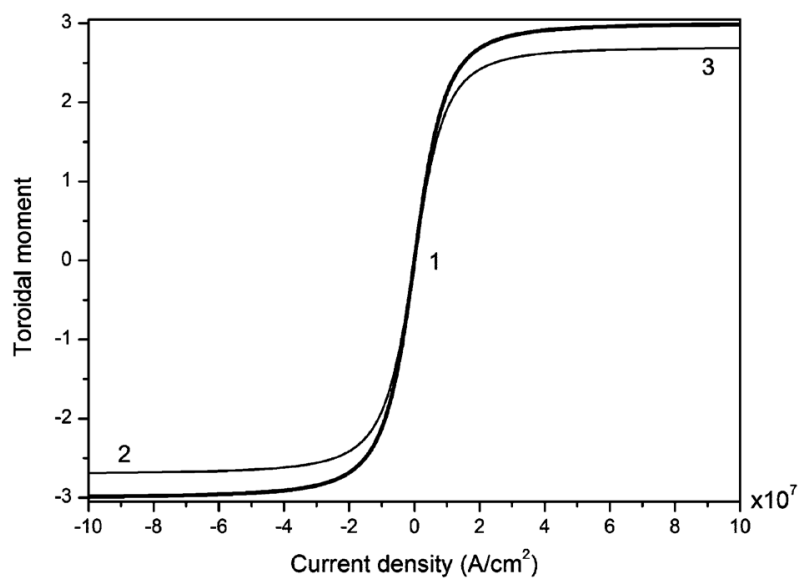

Figure 2. The zero temperature plot of toroidal moment projection onto the laboratory $Z$-axis vs the external current density: thicker curve (1) in equilibrium, the other curves in the case of the finite value of current sweeping rate $10^{13} \mathrm{~A} /\left(\mathrm{cm}^{2} \cdot \mathrm{s}\right)$, (2) from $\tau=+3$ to $\tau=-2.7$ and (3) from $\tau=-3$ to $\tau=+2.7$.

The tunneling processes when sweeping the current $j_{z}$ at a constant rate over an avoided energy level crossing can be treated in the frames of the Landau-Zener tunneling model. The probability $P$ to change the state characterized by the quantum number of the toroidal moment at the avoided level crossing is given by the expression

$$
P=1-\exp \left(-\frac{\pi \delta^{2}}{(4 \pi / c) \hbar \cdot T_{0} \cdot\left|\tau_{1}-\tau_{2}\right| \cdot\left|d j_{z} / d t\right|}\right),
$$

where $\tau_{1,2}= \pm 3$ are the toroidal moment quantum numbers of the avoided level crossing with the splitting $\delta$, and $d j_{z} / d t$ is the current sweeping rate. For $d j_{z} / d t \sim 10^{13}$ $\mathrm{A} /\left(\mathrm{cm}^{2} \cdot \mathrm{s}\right)$ and $\Delta / j=0.005$ we have $P \approx 0.95$.

With the Landau-Zener-Stückelberg model in mind, we can now start to understand qualitatively the hysteresis in the system considered. If current $j_{z}$ is sufficiently large negative, than, at very low temperature, all molecules are in the $|\tau=-3\rangle$ ground state. When the current is come down to zero, all molecules will stay in the $|\tau=-3\rangle$ ground state. When passing the current over the avoided level crossing region at $j_{z} \approx 0$, there is Landau-Zener probability $P(0<P<1)$ to tunnel from the $|\tau=-3\rangle$ to the $|\tau=+3\rangle$ state. The dependence of the toroidal moment on the current then reads as follows

$$
\tau\left(x, j_{z}\right)=\frac{c}{4 \pi T_{0}}\left(P \cdot \frac{\partial E_{+}\left(x, j_{z}\right)}{\partial j_{z}}+(1-P) \cdot \frac{\partial E_{-}\left(x, j_{z}\right)}{\partial j_{z}}\right)
$$


shown in fig. 2 (curve 3). The toroidal moment undergoes the jump of $\Delta \tau=6 P$. So the average value of toroidal moment $\tau$ becomes less than 3, namely $\tau=2.7$ for $P=0.95$. The relevant mixed quantum state relaxes to the equilibrium $|\tau=+3\rangle$ state due to interaction with environment (the $3 \rightarrow 1$ process in fig. 2).

Sweeping the current in the opposite direction from the $|\tau=+3\rangle$ state, we arrive to the mixed state with toroidal moment average value of -2.7 , see curve 2 in fig. 2 . The state relaxes as above to the equilibrium $|\tau=-3\rangle$ state (the $2 \rightarrow 1$ process in fig. 2). This way, we will come to the closed hysteresis loop.

\section{Anapole qubit}

In this section, we will briefly discuss the possibilities of using single-molecule toroics as qubits in quantum computing. It was shown, that the most important characteristic of such systems is the toroidal (anapole) moment, which is effectively coupled with electric current, therefore we will call such a qubit an anapole one.

1. The first requirement of creating systems of many $\left(N>10^{3}\right)$ qubits, each of which has two discrete energy levels, can easily be fulfilled. Indeed, all the molecules are obviously identical and can be synthesized in any necessary amount. What levels can be working ones in the qubit? To answer the question, suppose that the molecules are subjected to an electric current. The electric current, generally speaking, depends on time, which is necessary for controlling the particle states during initialization and when performing logical operations.

We will focus on the states $\left|T_{Z}=+3 T_{0}\right\rangle$ and $\mid T_{Z}=$ $\left.-3 T_{0}\right\rangle$, denoting them $|\uparrow\rangle$ and $|\downarrow\rangle$ accordingly. All other states have a much higher energy than those indicated if the electric current is not too strong. In this case the system can be considered a two-level system, and its Hamiltonian, written in the $\frac{1}{2}$-spin representation, has the simple form

$$
\mathcal{H}=-\frac{\varepsilon}{2} \sigma_{z}-\frac{\delta}{2} \sigma_{x}=-\frac{E}{2}\left(\sigma_{z} \cdot \cos \varphi+\sigma_{x} \sin \varphi\right),
$$

where $\varepsilon=\frac{4 \pi}{c} \cdot 3 T_{0} \cdot j_{z}, \delta$ is the avoided level splitting for zero current (see the previous section), $E=\sqrt{\varepsilon^{2}+\delta^{2}}$, and $\tan \varphi=\delta / \varepsilon$. The eigenstates of the Hamiltonian are the logical states of the qubit:

$$
\begin{gathered}
|0\rangle=\cos \frac{\varphi}{2}|\uparrow\rangle+\sin \frac{\varphi}{2}|\downarrow\rangle, \\
|1\rangle=-\sin \frac{\varphi}{2}|\uparrow\rangle+\cos \frac{\varphi}{2}|\downarrow\rangle .
\end{gathered}
$$

2. The second requirement to the sterling qubit deals with the possibility of preparing qubits of a quantum computer in the ground state $|00 \ldots 0\rangle$. Obviously, initialization can be achieved only with such deep cooling of an array of qubits, in which thermal excitations can not cause transitions to the higher state. This implies the natural requirement for an interval of operating temperatures $T<<j / k_{B} \sim 15 \mathrm{~K}$. Of course, achieving such low temperatures is not an easy task, and it should be noted that the vast majority of qubit implementations have this drawback, but the limitation on the maximum possible temperature in the case of single-molecule toroics can be weakened by using molecules with larger interionic exchange constant. At the same time, this operating temperature range is three orders wider than that of traditional magnetic particles using the magnetic moment as the degree of freedom. Thus, the anapole qubit has an indisputable advantage.

3. The next requirement concerns the possibility of overcoming the relaxation of the degrees of freedom used in the qubit (decoherence). During the execution of calculations (unitary transformation), the qubits of the computer are constantly exposed to the environment. As a result, the amplitude and phase of the state vector of each qubit experience unwanted changes thus randomness acquires after the decoherence time has elapsed. However, it is possible to achieve a stable quantum computing process for an arbitrarily long period of time if the methods of phase and amplitude error correction are used. It is proved [17] that with relatively low requirements for error-free execution of elementary operations (the probability of error is not more than $10^{-4}$ per computational step), quantum correction methods ensure stable operation.

The calculation of the decoherence time is a non-trivial task, the solution of which is far beyond the scope of this paper. However, here we can make some numerical estimates. As we shall see, the execution time of one step of computation is the order of $\Delta t=10 \mu \mathrm{s}$. This means that the decoherence time required for a stable quantum computing process must be at least $\tau_{c o h}=10^{4} \cdot \Delta t \sim 100 \mathrm{~ms}$. According to ref. [18], the dysprosium based molecular clusters demonstrate the slow relaxation behavior with comparable lifetime of the ground states limited by the macroscopic quantum tunneling at $5 \mathrm{~K}$.

4. The fourth requirement concerns the feasibility of the basic logic elements (gates) on qubits of this type. It is proved [21] that a quantum computer can be constructed from gates of only two types: a single-qubit gate and the two-qubit CNOT (controlled NOT). It should be noted that almost any logically invertible two-qubit gate (the exception is the classical SWAP gate) in combination with onequbit gates allows performing any predetermined computational algorithm. The selection of the CNOT element is owed to the simplicity of its realization.

Suppose that the anapole qubit is initially in a state far from the degeneracy point. In this case, the eigenstates $|0\rangle$ and $|1\rangle$ are almost the states $|\uparrow\rangle$ and $|\downarrow\rangle$, respectively. The sudden pulse of an electric current forces the system to arrive in the point of degeneracy. Then, a rotation occurs in the effective spin space under the influence of the operator

$$
U_{x}(\alpha)=\exp \left(\frac{i \alpha}{2} \sigma_{x}\right), \quad \alpha=\frac{\delta \Delta t}{\hbar},
$$

after which the backward pulse of the electric current dropping to zero removes the system from the degeneracy point. With this sequence of operations, the logical operation NOT corresponding to a rotation through angle $\alpha=\pi$ produced over a period of time $\Delta t=\frac{\pi \hbar}{\delta} \sim 10 \mu \mathrm{s}$. 
The phase shift between two logical states can be achieved by varying the magnitude of the electric current for a certain time by a small amount. The corresponding operator has the form

$$
U_{z}(\beta)=\exp \left(\frac{i \beta}{2} \sigma_{z}\right), \quad \beta=\frac{E_{0} \Delta t}{\hbar} .
$$

The CNOT valve is executed by acting on two interacting qubits: by means of the interaction, one qubit controls the evolution of the other. There exists a weak interaction between the qubits. However, when carrying out calculations on a quantum computer, it is desirable to have a controlled coupling between different qubits. Therefore, the coupling must be organised artificially. Without specifying yet the nature of the coupling between the qubits, we point out that the Ising type interaction $\left(H_{i n t}^{(1,2)}=J^{(1,2)} \sigma_{z}^{(1)} \sigma_{z}^{(2)}\right)$ during the following sequence of state transformations of the 1st (controlling) and 2nd (controlled) qubits leads to the execution of the CNOT gate:

$$
\begin{aligned}
& H^{(2)} \cdot U_{z}^{(1)}\left(\frac{\pi}{2}\right) \cdot U_{z}^{(2)}\left(\frac{\pi}{2}\right) \cdot U^{(1,2)}\left(\frac{\pi}{2}\right) \cdot H^{(2)}, \\
& U^{(1,2)}(\phi)=\exp \left(-i \phi \sigma_{z}^{(1)} \sigma_{z}^{(2)}\right), \quad \phi=\frac{J \Delta t}{\hbar} .
\end{aligned}
$$

In this sequence, the operator $H^{(2)}$ performs the Hadamard transformation,

$$
H^{(2)}=U_{x}^{(2)}\left(\frac{\pi}{2}\right) \cdot U^{(2)}\left(\frac{\pi}{2}\right) \cdot U_{x}^{(2)}\left(\frac{\pi}{2}\right) .
$$

5. Finally, the problem of measuring the states of qubits at the end of the computational process emerges. This, in fact, is the weakest point in almost all known qubit implementations. Unfortunately, there are no mastered technologies for such measurements.

The problem is closely related to the problem of controlling their states, which can be solved by the aid of superconductive current loops surrounding SMT particles. Electromagnetic signals coming from the computercontrolled device change the current in the loops, thereby generating an unsteady electric fields resulting in the displacement currents necessary to perform single-qubit operations. Using an additional system of current loops equipped with Josephson switches, a controlled interaction between any two qubits can be organized. The same loops can be used to measure the states of magnetic particles at the end of the calculations.

This scheme is aided by the effective coupling between current and toroidal moment, and has a number of advantages. First, the current loops have a small value of the power of the dissipated heat energy, which obviously favors the maintenance of the quantum coherence of the qubit states in the calculations. Secondly, the Josephson switches available in the circuit are capable of switching in a very short time.

\section{Conclusion}

The analysis of the toroidal moment dynamics gives the opportunity to assert that the electric current is an effective means of controlling the states of the ring rareearth molecular nanoclusters. Thus, the toroidal moment appears to be a promising versatile degree of freedom for quantum computing. The rare-earth single molecule toroics are considered to be a possible physical realization of a qubit as they meet the requirements of DiVincenzo.

We would like to acknowledge the financial support of Russian Foundation for Basic Research (projects 15-0208509 and 16-29-14005).

\section{References}

[1] R. Feynman, Inter. Jour. Theor. Phys. 21, 467 (1982).

[2] P. W. Shor, Proc. of the 35th Annual Symposium on the Foundations of Computer Science, 1994, p. 124.

[3] D. P. DiVincenzo, Fortschritte der Physik, 48, 771 (2000), see also arXiv:quant-ph/0002077.

[4] H. Walther, B. T. H. Varcoe, B.-G. Englert, T. Becker, Rep. Prog. Phys. 69, 1325 (2006).

[5] P. Kok, W. J. Munro, K. Nemoto et al., Rev. Mod. Phys. 79, 135 (2007).

[6] S. Aaronson and A. Arkhipov, Theory of Computing 9, 143 (2013).

[7] D. Kielpinski, C. Monroe, and D. J. Wineland, Nature 417, 709 (2002).

[8] G. K. Brennen, C. Caves, P. S. Jessen, and I. H. Deutsch, Phys. Rev. Lett. 82, 1060 (1999).

[9] B. E. Kane, Nature 393, 133 (1998); J. O'Gorman et al., Nat. Phys. J. Quant. Inf. 2, 15019 (2016).

[10] D. Loss and D. P. DiVincenzo, Phys. Rev. A 57, 120 (1998).

[11] L. Gordon, J. R. Weber, J. B. Varley et al., MRS Bulletin 38, 802 (2013).

[12] C. Rigetti, J. M. Gambetta, S. Poletto et al., Phys. Rev. B 86, 100506 (2012).

[13] J. Tejada, E. M. Chudnovsky, E. del Barco et al., Nanotechnology 12, 181 (2001).

[14] A. K. Zvezdin and D. I. Plokhov, Bulletin of the Lebedev Physics Institute, issue 12 (2003), in Russian.

[15] L.F. Chibotaru, L. Ungur, and A. Soncini, Angew. Chem. Int. Ed. 47, 4126 (2008).

[16] D.I. Plokhov, A.K. Zvezdin, and A.I. Popov, Phys. Rev. B 83, 184415 (2011).

[17] A. M. Steane, Rep. Progr. Phys. 61, 117 (1998).

[18] P.-H. Guo, J.-L. Liu, Z.-M. Zhang et al., Inorg. Chem. 51, 1233 (2012).

[19] A. Barenco, C. H. Bennett, C. Cleve et al., Phys. Rev. A 52, 3457 (1995). 